\title{
L'infertilité masculine: Nos connaissances ne doivent pas faire oublier notre ignorance
}

\author{
Thierry BIENVENU \\ INSERM U567, Institut Cochin, Université Paris V et Laboratoire de Biochimie et Génétique Moléculaire, Hôpital \\ Cochin, AP-HP, Paris, France
}

\section{RESUME}

Environ $15 \%$ des couples dans le monde sont concernés par des problèmes de procréation. Dans $20 \%$ des cas, c'est l'homme seul qui est en cause. Dans $20 \%$ de ces cas, une cause génétique peut être identifiée. Les principales causes peuvent se classer en trois grandes catégories : i) les anomalies chromosomiques, ii) les microdélétions du bras long du chromosomes $Y$ et iii) les mutations du gène CFTR. Toutefois, malgré les progrès considérables réalisés au cours de ces dernières années, la cause de l'infertilité est ignorée dans un grand nombre de cas. Le développement des stratégies gène candidat, les analyses de liaison génétique dans des formes familiales d'infertilité masculine, la création de modèles animaux ainsi que les études du remodelage de la chromatine au cours de la spermiogénèse devraient permettre d'identifier à court terme de nouveaux facteurs génétiques, qui chez l'homme sont à l'origine de stérilité.

Mots Clés : infertilité masculine, microdélétion du chromosome $Y$, anomalies chromosomiques, remodelage de la chromatine
Environ $15 \%$ des couples dans le monde sont concernés par des problèmes de procréation. Dans $20 \%$ des cas, c'est l'homme qui est en cause et dans près de $30 \%$ des cas les deux partenaires sont en cause [15]. Les travaux scientifiques réalisés au cours de ces 20 dernières années ont permis d'identifier une cause génétique dans environ $20 \%$ des cas d'infertilité masculine. Ces causes peuvent se classer en trois grandes catégories : i) les anomalies chromosomiques, ii) les microdélétions du bras long du chromosomes $\mathrm{Y}$ et iii) les mutations du gène CFTR [6].

Des remaniements chromosomiques sont en effet identifiés chez près de $6 \%$ des hommes infertiles ( $13 \%$ chez les hommes azoospermiques) [3]. Il peut s'agir d'anomalies affectant les chromosomes sexuels, incluant notamment le syndrome de Klinefelter $(47, \mathrm{XXY})$ qui représente plus de $60 \%$ des anomalies de ce type, et d'anomalies affectant la structure des autosomes. Tous les types de remaniements chromosomiques ont été décrits dans les cas d'infertilité masculine : translocations robertsoniennes (fusion centrique des chromosomes acrocentriques), translocations réciproques, inversions paracentriques. Le remaniement chromosomique peut supprimer l'expression d'un ou de plusieurs gènes impliqués dans le spermatogénèse ou empêcher le bon appariement des chromosomes homologues (asynapsis) déclenchant un arrêt de la méiose. Le plus souvent, les anomalies de nombre des chromosomes sexuels sont associées à une azoospermie, alors que les anomalies de structure des autosomes sont associées à une oligozoospermie.

Des délétions interstitielles du bras long du chromosome

Correspondance :

Dr Thierry Bienvenu - Laboratoire de Biochimie et génétique Moléculaire. Hôpital Cochin, 123 boulevard de Port-Royal., 75014 Paris, France - Tel 01.44.41.24.79 - Fax 01.58.41.15.80 - Email bienvenu@cochin.inserm.fr 
Y (Yq11.23) sont retrouvées chez environ $7 \%$ des hommes infertiles [9]. Ces délétions le plus souvent de novo peuvent affecter une ou plusieurs régions du bras long du chromosome $\mathrm{Y}$ (dénommées $\mathrm{AZFa}, \mathrm{AZFb}$ et $\mathrm{AZFc}$ ) [14]. Bien qu'hétérogènes en terme de localisation et de taille, ces délétions sont retrouvées essentiellement chez des sujets azoospermiques ou oligozoospermiques sévères (< 0,1 million de spermatozoïdes / $\mathrm{mL}$ ) [10].

Des mutations ponctuelles localisées dans le gène CFTR permettent d'expliquer la grande majorité des cas d'azoospermie obstructive par absence bilatérale des canaux déférents $(A B C D)$ [8]. Ce type d'azoospermie représente près de $25 \%$ des azoospermies obstructives et ainsi près de $2 \%$ des infertilités masculines. L'analyse de la totalité de la région codante du gène $C F T R$ permet d'identifier une mutation au moins chez environ $87 \%$ des sujets $A B C D$ [1]. Dans $71 \%$ des cas, les sujets sont homozygotes ou hétérozygotes composites. Ils présentent sur une des copies du gène CFTR une mutation dite modérée et sur l'autre copie soit une mutation sévère soit une mutation modérée. L'absence d'identification d'une mutation qui peut échapper aux méthodes d'analyses réalisées en routine par les laboratoires de génétique moléculaire ne signifie pas que cette mutation soit modérée. Certaines mutations rares sont en effet des mutations sévères.

Ainsi, la prescription d'un caryotype chez un homme infertile, d'une recherche de microdélétions du chromosome $Y$ notamment chez les hommes azoospermiques ou oligozoospermiques sévères, et d'une recherche de mutations dans le gène $C F T R$ en cas d'absence bilatérale des canaux déférents doit permettre d'identifier une cause à l'infertilité dans près de $20 \%$ des cas et à l'azoospermie dans près de $60 \%$ des cas.

Le recours à la procréation médicalement assistée pour un grand nombre de ces couples dont l'homme est infertile implique de fournir au couple un conseil génétique expliquant les risques de récurrence de la maladie ou de survenue de maladies génétiques plus graves et de les orienter vers la solution la plus adaptée à leur problème. C'est à ce stade que des analyses génétiques complémentaires (comme par exemple l'étude des spermatozoïdes par hybridation in situ de sondes fluorescences en cas de remaniement chromosomique) et l'analyse moléculaire de l'ADN de la conjointe doivent être envisagées. Ainsi par exemple si un homme présente une $\mathrm{ABCD}$, la recherche de mutations dans le gène CFTR doit être réalisée chez sa conjointe afin de calculer le risque pour ce couple d'avoir un enfant atteint d'une ABCD ou d'une mucoviscidose. Par ailleurs, l'analyse génétique peut avoir un intérêt pronostique. Ainsi la probabilité de trouver des spermatozoïdes à la biopsie testiculaire est pratiquement nulle chez les hommes ayant une azoospermie et une microdélétion affectant
$A Z F a$ ou englobant $A Z F c$ et d'autres régions $(A Z F b+c$, $A Z F a+b+c)$, ce qui conditionne la prise en charge ultérieure de ce couple.

Toutes ces récentes acquisitions scientifiques ne doivent toutefois pas masquer notre ignorance sur les causes de l'infertilité de l'homme dans un grand nombre de cas. Il reste ainsi probablement un grand nombre de gènes impliqués dans la survenue d'une infertilité à identifier. Trois approches sont ainsi développées :

i) l'approche gène candidat qui consiste à choisir des gènes de séquence connue, hautement conservés au cours de l'évolution des espèces, facile à analyser et de préférence dont l'invalidation (knock-out) chez la souris aboutit à une stérilité des mâles. Cette approche a été récemment appliquée pour 5 gènes de méiose (DCM1, SPO11, MSHA, MSH5, CCNA1) et a permis d'identifier chez des hommes infertiles des variants dont les conséquences sur la méiose sont en cours d'étude chez la levure [5];

ii) l'approche génétique qui consiste, dans des formes familiales d'infertilité masculine, à rechercher une liaison génétique entre un marqueur polymorphe localisé dans un territoire génomique connu et la maladie. C'est cette approche qui a permis récemment d'impliquer le gène CATSPER2 localisé sur le bras long du chromosome 15 (15q15) dans certaines formes d'infertilité masculine [5] ;

iii) l'approche basée sur le développement de modèles animaux. Au cours de ces dernières années, l'utilisation de la transgénèse chez la souris a permis de démontrer le rôle de certains gènes soupçonnés d'intervenir dans la spermatogénèse comme par exemple PMS2, MLH1, BAX, CREM, HSP70-2, RXRM, Dhh et plus récemment eIF2 [2, 5]. Toutefois, la mise en cause d'un de ces gènes dans des cas d'infertilité humaine masculine n'a pas encore été obtenue.

Pour encore mieux comprendre la difficulté de la tache, il ne faut pas oublier que l'ADN du génome est complexé avec des protéines de petite taille, de 11 à $14 \mathrm{kDa}$ et fortement basiques, les histones. Au cours de la spermiogénèse, ces protéines sont remplacées par des protéines de transition (TP1 et TP2), puis finalement par les protamines. Ce changement permet le remodelage de la chromatine et une forte condensation de l'ADN dans le spermatozoïde mature. Cette condensation est précédée par une hyperacétylation des histones $[11,12]$. Ce phénomène concerne 13 résidus lysine des histones $\mathrm{H} 2 \mathrm{~A}, \mathrm{H} 2 \mathrm{~B}, \mathrm{H} 3$ et $\mathrm{H} 4$. Ce sont les histones acétyltransférases (HAT) et les histones déacétylases qui contrôlent respectivement les mouvements d'acétylation et de déacétylation des histones [7]. Une perturbation de cet équilibre se traduit par des modifications d'affi- 
nité des histones pour l'ADN (ou des histones entre elles) et donc par des modifications de l'accessibilité de régulateurs transcriptionnels à l'ADN. Des perturbations de cet équilibre ont ainsi été mis en évidence chez des hommes infertiles au niveau des spermatides [13], mais aussi au niveau des cellules de Sertoli dont les interactions avec les cellules germinales sont primordiales [2, 12]. Ainsi, les gènes intervenant dans le remodelage de la chromatine (comme par exemple $C D Y$ localisé sur le bras long du chromosome $\mathrm{Y}$ ou BRDT (bromodomain-containing protein)) ainsi que les gènes exprimés grâce à ce phénomène dans les cellules postméiotiques sont des candidats potentiels pour les cas d'infertilité masculine. Reste maintenant à les identifier...

\section{REFERENCES}

1. CLAUSTRES M., GUITTARD C., BOZON D. et al. : Spectrum of CFTR mutations in cystic fibrosis and in congenital absence of the vas deferens in France. Hum. Mutat., 2000, $16: 143-156$.

2. COOKE H.J., SAUNDERS P.T.K. : Mouse models of male infertility. Nature Rev., 2002, $3: 790-801$.

3. DAHOUN S. : Anomalies chromosomiques et infertilité masculine. XIX Congrès de la société d'andrologie de langue française. 12-14 décembre 2002, Genève, Suisse.

4. ESCALIER D. : Impact of genetic engineering on the understanding of spermatogenesis. Hum. Reprod. Update, 2001, 7 : 191210.

5. FELLOUS M. : Modèles d'étude et perspectives. XIX Congrès de la société d'andrologie de langue française. 12-14 décembre 2002, Genève, Suisse.

6. HUYNH T., MOLLARD R., TROUNSON A. : Selected genetic factors associated with male infertility. Hum. Reprod. Update, 2002, $8: 183-198$.

7. LAHN B.T., LAN TANG Z., ZHOU J. et al. : Previously uncharacterized histone acetyltransferases implicated in mammalian spermatogenesis. Proc. Natl. Acad. Sci. U.S.A., 2002, 99 : 8707 8712.

8. LAYMAN L.C. : Human gene mutations causing infertility. J. Med. Genet., 2002, 39 : 153-161.

9. MORRIS M. : Anomalies géniques et infertilité masculine. XIX Congrès de la société d'andrologie de langue française. 12-14 décembre 2002, Genève, Suisse.

10. PATRAT C., BIENVENU T., JOUANNET P. : Microdélétions du chromosome $\mathrm{Y}$ et infertilité masculine. Ref. Gynecol. Obstet., 2001, $8: 13-20$.

11. RICE J.C., ALLIS C.D. : Histone methylation versus histone acetylation : new Insights into epigenetic regulation. Curr. Opin. Cell Biol., 2001, 13 : 263-273.

12. ROUSSEAU S. : Facteurs impliqués dans le remodelage de la chromatine au cours de la spermiogénèse. XIX Congrès de la société d'andrologie de langue française. 12-14 décembre 2002, Genève, Suisse.

13. SONNACK V., FAILING K., BERGMANN M., STEGER K. :
Expression of hyperacetylated histone $\mathrm{H} 4$ during normal and impaired human spermatogenesis. Andrologia, 2002, 34 : 384390.

14. VOGT P.H., EDELMANN A., KIRSCH S, et al. : Human Y chromosome azoospermia factors (AZF) mapped to different subregions in Yq11. Hum. Mol. Genet., 1996, 5 : 933-943.

15. World Health Organization : Towards more objectivity in diagnosis and management of male infertility. Int. J. Androl., 1987, 7, suppl. : 1-53.

Male infertility: our recent acquisition of knowledge must not conceal our ignorance

Thierry BIENVENU

About $15 \%$ of couples worldwide are affected by reduced fertility. In $20 \%$ of cases of couple infertility, the problem can be predominantly attributed to the male. In $\mathbf{2 0 \%}$ of cases, a genetic cause of male infertility can usually be identified. The main genetic causes are: autosomal and sex chromosomal abnormalities, microdeletions within regions of the Y-chromosome containing candidate gene families for spermatogenesis and mutations in the CFTR gene. However, despite enormous progress in the understanding of human reproductive physiology, the underlying cause of male infertility often cannot be elucidated. Candidate gene strategies, linkage analysis in large familial forms of male infertility, targeted mutagenesis in the mouse and studies of chromatin reorganization during spermatid maturation should provide rapid progress in our understanding of the genetic factors that contribute to male infertility, which may open up new approaches to the treatment of this condition.

Key-Words: male infertility, Y-chromosome microdeletion, chromosome abnormalities, genome repackaging 